\title{
Crystal Structure of the Archaeosine Synthase QueF-Like - Insights into Amidino Transfer and tRNA Recognition by the Tunnel Fold
}

\author{
Xianghan Mei ${ }^{1, \#}$, Jonathan Alvarez ${ }^{2, \#}$, Adriana Bon Ramos ${ }^{3}$, Uttamkumar Samanta $^{2}$, Dirk \\ Iwata-Reuyl ${ }^{3}$, and Manal A. Swairjo ${ }^{1, *}$ \\ ${ }^{1}$ Department of Chemistry and Biochemistry, San Diego State University- 5500 Campanile Drive, \\ San Diego, CA 92182 \\ ${ }^{2}$ Graduate College of Biomedical Sciences, Western University of Health Sciences, Pomona, CA \\ 91766-1854 \\ ${ }^{3}$ Department of Chemistry, Portland State University, PO Box 751, Portland, OR 97207
}

\begin{abstract}
The tunneling-fold (T-fold) structural superfamily has emerged as a versatile protein scaffold of diverse catalytic activities. This is especially evident in the pathways to the 7-deazaguanosine modified nucleosides of tRNA queuosine and archaeosine. Four members of the T-fold superfamily have been confirmed in these pathways and here we report the crystal structure of a fifth enzyme; the recently discovered amidinotransferase QueF-Like (QueF-L), responsible for the final step in the biosynthesis of archaeosine in the D-loop of tRNA in a subset of Crenarchaeota. QueF-L catalyzes the conversion of the nitrile group of the 7-cyano-7-deazaguanine ( $\mathrm{preQ}_{0}$ ) base of pre $\mathrm{Q}_{0}$-modified tRNA to a formamidino group. The structure, determined in the presence of pre $_{0}$, reveals a symmetric T-fold homodecamer of two head-to-head facing pentameric subunits, with 10 active sites at the inter-monomer interfaces. Bound $\mathrm{preQ}_{0}$ forms a stable covalent thioimide bond with a conserved active site cysteine similar to the intermediate previously observed in the nitrile reductase QueF. Despite distinct catalytic functions, phylogenetic distributions, and only $19 \%$ sequence identity, the two enzymes share a common pre $\mathrm{Q}_{0}$ binding pocket, and likely a common mechanism of thioimide formation. However, due to tight twisting of its decamer, QueF-L lacks the NADPH binding site present in QueF. A large positively charged molecular surface and a docking model suggest simultaneous binding of multiple tRNA molecules and structure-specific recognition of the D-loop by a surface groove. The structure sheds light on the mechanism of nitrile amidation, and the evolution of diverse chemistries in a common fold.
\end{abstract}

\section{Keywords}

Tunneling-fold enzyme; T-fold; 7-deazaguanosine; QueF-L; 7-cyano-7-deazaguanine; pre $_{0}$; thioimide; amidinotransferase; transfer-RNA; modified nucleoside

\footnotetext{
*Corresponding author. mswairjo@mail.sdsu.edu.

\#these authors contributed equally to this work.
} 


\section{INTRODUCTION}

The 7-deazaguanosine nucleosides queuosine $(\mathrm{Q})$ and archaeosine $\left(\mathrm{G}^{+}\right)$are the most remarkable modifications known to occur in tRNA (Fig. 1). While sharing a common 7deazaguanosine core, and a significant portion of their biosynthetic pathways, they differ in structure, their location on tRNA, and their phylogenetic distribution. Q is characterized by a cyclopentenediol ring appended to an aminomethyl group at the 7-position ${ }^{1,2}$ (which may be glycosylated with galactose or mannose, ${ }^{3}$ or acylated with glutamate ${ }^{4,5}$ in some species). $\mathrm{G}^{+}$possesses an amidine functional group at the 7-position. ${ }^{6} \mathrm{Q}$ is found in the tRNA of Bacteria and Eukarya at position-34 (the wobble position) in tRNAs decoding NAC/U codons, ${ }^{7}$ while $\mathrm{G}^{+}$is found only in Archaea at position- 15 in the dihydrouridine loop (Dloop). ${ }^{8}$ Consistent with its position in the anticodon, Q plays a role in modulating codon anticodon binding efficiency,,${ }^{9} 10$ while the presence of the positively charged formamidine group of $\mathrm{G}^{+}$is believed to be important in stabilization of the tertiary structure of tRNA through electrostatic interactions with the anionic phosphates and by enhancing the hydrogen bonding within the G15-C48 Levitt base pair. ${ }^{11}$

The biosynthetic pathways to $\mathrm{Q}$ and $\mathrm{G}^{+}$have been fully elucidated only recently. The core of $\mathrm{Q}$ and $\mathrm{G}^{+}$is biosynthesized from GTP by the action of GTP cyclohydrolase $\mathrm{I},{ }^{12}$ followed by the QueD, QueE, and QueC enzymes to produce the advanced intermediate 7-cyano-7deazaguanine $\left(\mathrm{preQ}_{0}\right.$ ) in both Bacteria ${ }^{13,14}$ and $\operatorname{Archaea}^{15}$ (Fig. 1). In bacteria, preQ $\mathrm{Q}_{0}$ is first reduced to 7-aminomethyl-7-deazaguanine ( $\mathrm{preQ}_{1}$ ) by the nitrile reductase $\mathrm{QueF}^{16}$ before insertion in substrate tRNAs by the bacterial tRNA-guanine transglycosylase (bTGT) ${ }^{17}$. In Archaea, preQ $_{0}$ is inserted directly in tRNA by the archaeal TGT homolog (arcTGT), ${ }^{18,19}$ and then converted to $\mathrm{G}^{+}$by the amidinotransferase $\mathrm{ArcS}^{20}$ in the majority of Archaea.

The biosynthetic pathway to $\mathrm{Q}$ and $\mathrm{G}^{+}$is especially rich in enzymes belonging to the tunneling-fold (T-fold) superfamily, with three steps catalyzed by T-fold enzymes, GCYH IA and IB, ${ }^{21,22}$ QueD, ${ }^{23}$ and QueF. ${ }^{24,25} \mathrm{~T}$-fold proteins share a small domain (T-fold domain) that consists of an antiparallel $\beta$-sheet of four strands and two antiparallel $\alpha$ helices, between the second and third strands $(\beta \beta a \alpha \beta \beta)$, layered on the concave face of the $\beta$-sheet. The domains oligomerize to form a $\beta_{2 n} a_{n}$ barrel, and two barrels join head-to-head to form a tunnel-like center. Although exhibiting a low level of sequence identity, these proteins exhibit high tertiary and quaternary structural similarity, with active sites located at the interfaces between subunits.

The T-fold has emerged as a versatile protein scaffold that supports diverse catalytic activities, ${ }^{26}$ a fact exemplified by the T-fold enzymes involved in 7-deazaguanosine biosynthesis (Fig. 1). The first, GTP cyclohydrolase IA and $\mathrm{IB}^{22}$, catalyzes the conversion of GTP to 7,8-dihydroneopterin triphosphate $\left(\mathrm{H}_{2} \mathrm{NTP}\right)$, a step shared with the folic acid ${ }^{27}$ and biopterin pathways. ${ }^{28}$ QueD, which catalyzes the second step of the pathway, converts $\mathrm{H}_{2} \mathrm{NTP}$ to carboxytetrahydropterin $\left.\left(\mathrm{CPH}_{4}\right)\right)^{23,29}$ The third T-fold enzyme is QueF, an enzyme unique to bacteria and the $\mathrm{Q}$ branch of the pathway. QueF catalyzes the NADPHdependent reduction of the nitrile group of $\mathrm{preQ}_{0}$ to the amine of $\mathrm{preQ}_{1} \cdot{ }^{16,24}$ 
We previously reported that an archaeal homolog of QueF, QueF-Like (QueF-L), found in a subset of Crenarchaeota that lack ArcS, was capable of producing $\mathrm{G}^{+}$-modified tRNA when expressed in an E. coli $\Delta q u e F$ mutant. ${ }^{30}$ This observation suggested that QueF-L functioned as an amidinotransferase analogous to $\mathrm{ArcS}$, converting the nitrile group of $\mathrm{preQ}_{0}$-modified tRNA, or free preQ $Q_{0}$, to the formamidine group of $\mathrm{G}^{+}$(Fig. 1). Subsequently, we demonstrated that the purified recombinant QueF-L from Pyrobaculum calidifontis does function as an amidinotransferase, and converts preQ $0_{0}$-modified tRNA, but not preQ $_{0}$, to $\mathrm{G}^{+}$modified tRNA (D. Iwata-Reuyl, manuscript in preparation). Notably, like QueF, QueF-L possesses an active-site cysteine that serves as a catalytic nucleophile, reacting with the nitrile group to form a covalent thioimide intermediate (D. Iwata-Reuyl, manuscript in preparation). Furthermore, although preQ $_{0}$ is not turned over by QueF-L, it does bind and react to form a covalent thioimide intermediate. In QueF the thioimide intermediate is the substrate for the first round of reduction with NADPH, while in QueF-L the thioimide intermediate formed with pre $_{0}$-tRNA is the substrate for a reaction with ammonia.

As its name indicates, QueF-L is homologous to QueF, exhibiting 18-20\% sequence identity, and thus was predicted to be a member of the T-fold superfamily. Here we describe crystal structures of QueF-L from the hyperthermophilic crenarchaeon Pyrobaculum calidifontis in the presence and absence of $\mathrm{pre}_{0}$, which confirm the predicted T-fold architecture and provide evidence for the structural basis of its functional divergence from QueF. QueF-L is the only example of amidinotransferase chemistry and tRNA binding function in the tunneling-fold structural superfamily, and represents a new class of amidinotransferases. QueF-L, together with the other two archaeosine synthases ArcS and GAT-QueC, are the only examples in Biology of the conversion of a nitrile to a formamidino group.

\section{MATERIALS AND METHODS}

\section{Over-production and purification of native and selenomethionine-labeled $\boldsymbol{P}$. calidifontis QueF-L}

Recombinant $P$. calidifontis QueF-L was over-produced in $E$. coli BL21(DE3) cells from a pET30 construct as an N-terminal His 6 -tagged protein possessing a Factor Xa cleavage site. Cells transformed with the overexpression vector were grown in LB/Kan medium to an $\mathrm{OD}_{600}$ of 0.9 before induction with addition of IPTG to final concentration of $0.25 \mathrm{mM}$ and aerobic growth at $37^{\circ} \mathrm{C}$ for 4 hours. The cells were collected by centrifugation at 7,500 $g$ for 15 minutes and re-suspended to a density of $250 \mathrm{mg} / \mathrm{mL}$ in $50 \mathrm{mM}$ Tris- $\mathrm{HCl}$ (pH 8.0), 300 $\mathrm{mM} \mathrm{KCl}, 2 \mathrm{mM} \beta \mathrm{ME}$, and $1 \mathrm{mM}$ PMSF (lysis buffer). Lysozyme was added to a final concentration of $250 \mu \mathrm{g} / \mathrm{mL}$ and the cells incubated at $37{ }^{\circ} \mathrm{C}$ for 30 minutes, followed by 3 intervals of freeze-thaw cycles. DNase was added to a final concentration of $10 \mu \mathrm{g} / \mathrm{mL}$ and the cells were left at $37^{\circ} \mathrm{C}$ for an additional 30 minutes. The cell lysate was centrifuged at $26,000 \mathrm{~g}$ for 30 minutes, and the cleared lysate was heated to $65^{\circ} \mathrm{C}$ for 15 minutes and centrifuged at $26000 \mathrm{~g}$ for 20 minutes. The resulting solution was filtered before loading onto $\mathrm{Ni}^{2+}$-NTA resin equilibrated in $50 \mathrm{mM}$ Tris- $\mathrm{HCl}(\mathrm{pH} 8.0), 300 \mathrm{mM} \mathrm{KCl}, 2 \mathrm{mM} \beta \mathrm{ME}$ (equilibration buffer). The column was washed with 5 column volumes of equilibration buffer followed by 5 column volumes of equilibration buffer with $20 \mathrm{mM}$ imidazole. The 
recombinant protein was eluted with 7 column volumes of equilibration buffer containing $200 \mathrm{mM}$ imidazole, then concentrated to about $2 \mathrm{~mL}$ (Amicon Ultra YM-10k) and dialyzed against equilibration buffer. The $\mathrm{His}_{6}$ tag was subsequently removed by Factor Xa cleavage and removed on Ni-NTA resin.

The SeMet-labeled protein was over-produced in the methionine auxotrophic E. coli strain B834(DE3) grown in minimal media supplemented with $0.05 \mathrm{mg} / \mathrm{mL}$ SeMet. ${ }^{31}$ Purification was carried out as described above. $80 \%$ incorporation of Se at the two Met residues in the polypeptide (including the $\mathrm{N}$-terminal Met) was verified by MALDI-TOF mass spectrometry.

\section{Crystallization}

Crystals of apo P. calidifontis QueF-L and of its complex with preQ $_{0}$ (synthesized as described $^{32}$ and purified via HPLC using a Luna C18 semi-preparative column (250 x 10 $\mathrm{mm}, 5$ micron) from Phenomenex (Torrance, $\mathrm{CA}$ ) with a mobile phase of ammonium acetate ( $25 \mathrm{mM}, \mathrm{pH}$ 6.0) and acetonitrile) were generated using the native protein and the SeMet labeled protein, respectively. For the complex crystals, preQ $_{0}$ was first added to a $4 \mathrm{mg} / \mathrm{mL}$

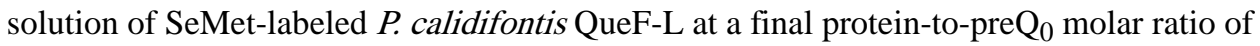
$1: 5$, and the mixture was incubated on ice for 30 minutes before crystallization. For both protein samples, crystals were produced using the vapor diffusion method and similar conditions. Briefly, for the native protein, hanging drops were set up by mixing $1 \mathrm{uL}$ of protein solution with $1 \mathrm{uL}$ of reservoir solution containing 17-19\% PEG3350, 0.15 M potassium thiocyanate, and $0.05 \%$ sodium azide, and equilibrated against $500 \mathrm{uL}$ of reservoir solution at $20^{\circ} \mathrm{C}$. For the SeMet-labeled protein in complex with $\mathrm{preQ}_{0}$, the same conditions were used, except crystallization was conducted in sitting drops set up by mixing $2 \mathrm{uL}$ of protein solution with $1 \mathrm{uL}$ of reservoir solution. Rod-shaped crystals $(0.25 \mathrm{~mm}$ long and $0.15 \mathrm{~mm}$ thick) appeared in 1-2 weeks and were directly cryo cooled in liquid nitrogen without need for a cryo protectant.

\section{X-ray Data collection and processing}

X-ray data collection was performed at the Stanford Synchrotron Research Laboratory beam line 7-1. First, the presence of the anomalous scatterer was verified in the SeMet crystals by $\mathrm{X}$-ray fluorescence using an excitation wavelength scan in the vicinity of the Se edge. A 3wavelength multiwavelength anomalous dispersion data set was collected at the peak and inflection energies of the Se edge, as well as at a remote energy below the Se edge at a detector-to-crystal distance of $350 \mathrm{~mm}$ and an oscillation angle of 0.5 degree. Data were processed in the HKL2000 program suite ${ }^{33}$ and data quality was checked in Xtriage. ${ }^{34}$ Data collection parameters and processing statistics are listed in Table 1.

\section{Crystal structure determination and refinement}

The crystal structure of the $\mathrm{pre}_{0}$-bound enzyme (space group $\mathrm{C} 2$ ) was determined by the multiwavelength anomalous dispersion method using $\mathrm{Se}$ as the anomalous scatterer (SeMAD) and the PHENIX suite of programs, and refined initially using PHENIX_refine and followed by 5 rounds of refinement and solvent fitting using CCP4 Refmac $5^{35}$ and Coot. ${ }^{36}$ To verify the presence of bound ligands in the active sites, omit Fo-Fc and 2Fo-Fc electron 
density maps were generated and inspected for each site. The apo structure (space group $\mathrm{P} 2{ }_{1}$ ) was determined by molecular replacement using the Phaser-MR software ${ }^{37}$ and the protein component of the $\mathrm{preQ}_{0}$-bound structure as a search model. The structure was refined using Refmac5 with solvent fitting and map inspection done in Coot. For both structures, in the final round of refinement, TLS parametrization was applied treating the 15 monomers in the asymmetric unit as TLS bodies. The coordinates and the structure factors of QueF-L/preQ ${ }_{0}$ complex and of apo QueF-L have been deposited in the RCSB Protein Data Bank under accession codes 5JYX and 5K0P, respectively.

\section{Generation of the docking model}

Docking calculations were performed using the HADDOCK (High Ambiguity Driven biomolecular DOCKing) web server (version 2.2). ${ }^{38}$ The protein and RNA components used for docking were constructed as follows. For the protein, four adjacent subunits, two from each pentamer, were extracted from the apo QueF-L structure. This protein component contained two empty, open active sites. For the RNA component, the seven-nucleotide Dloop segment (residues A14-C21) of the $\lambda$-form tRNA was extracted from its crystal structure in complex with $P$. horikoshii archaeosine tRNA-guanine transglycosylase (PDB ID $1 \mathrm{~J} 2 \mathrm{~B}$ ), and the guanine base at position 15 was replaced with preQ $_{0}$ to make a model for a preQ $\mathrm{Q}_{0}$-modified D-loop. In HADDOCK, $\mathrm{preQ}_{0} 15$ in the D-loop and the residues defining the preQ $_{0}$ binding pocket in QueF-L (Cys21, Asp28, His62 and Glu63 from one subunit, and Leu43, Glu46 from the adjacent subunit) were set as "active residues" (residues that have been shown experimentally to be involved in binding). These interactions were the only restraints imposed on the docking search. 140 docking models were generated and grouped in 10 clusters that were ranked by their Haddock score. The top two clusters had comparable HADDOCK scores (the weighted sum of Van der Waals, electrostatic, de-solvation and restraint-violation energies) of -9.4 and -7.2 (the next cluster scored $\sim 0$ ). Based on visual inspection of the resulting models from both clusters, the final model was picked for best fit of the $\mathrm{preQ}_{0}$ base in the active site.

\section{Figures}

Figures in this article were made using the PyMOL Molecular Graphics System, Version 1.8 Schrödinger, LLC. (http://www.pymol.org).

\section{RESULTS AND DISCUSSION}

\section{Overall structure}

We determined two crystal structures of $P$. calidifontis QueF-L (109 residues, 12055 Dalton). First, the crystal structure of the enzyme bound to $\mathrm{preQ}_{0}$ (C2 space group) was determined by the selenium-multiwavelength anomalous dispersion (Se-MAD) method and refined to $2.74 \AA$ (Table 1 ). The apo structure ( $\mathrm{P} 2_{1}$ space group) was then solved by molecular replacement using a single protein subunit from the preQ $_{0}$-bound structure as a search model, and was refined to $1.91 \AA$. Although the two crystal forms contain a different number of protein subunits in the asymmetric unit ( 15 and 10 subunits in the $\mathrm{C} 2$ and $\mathrm{P} 2{ }_{1}$ crystal form, respectively), the biological assembly in both structures is the same. Each structure reveals a symmetric tunnel-fold homodecamer of two head-to-head facing 
pentameric subunits, harboring 10 active sites at the intermonomer interfaces (Fig. 2). Each pentamer is composed of a cyclic arrangement of monomeric subunits and exhibits C5 symmetry. The pentamers from both structures are similar and superpose with r.m.s.d $0.7 \AA$ over all $1010 C_{a}$ atoms. The monomer is a minimal, single T-fold $\beta 4 a 2$ domain constituted of a highly twisted, amphipathic, antiparallel $\beta$-sheet $(\beta 1, \beta 2, \beta 3, \beta 4)$ and two antiparallel $\alpha$ helices $(a 1, a 2)$ layered on its concave side (Fig. 2). The central tunnel of the decamer is 24 $\AA$ wide and $58 \AA$ long, and is lined by an extensive network of 60 salt bridges formed by conserved ionic side chains protruding into the tunnel from the hydrophilic face of the beta barrel (Fig. 2). Members of the salt-bridge pairs originate in adjacent beta strands within each monomeric subunit and in between subunits, conferring to the beta barrel additional structural stability presumably needed at the extremely high temperature environment (95$102^{\circ} \mathrm{C}$ ) of the host organism.

\section{QueF-L binds pre $_{0}$ and forms a thioimide intermediate}

In the structure obtained from the crystal grown in the presence of pre $_{0}$, clear electron density corresponding to $\mathrm{preQ}_{0}$ is seen in the $\mathrm{Fo}-\mathrm{Fc}$ and $2 \mathrm{Fo}-\mathrm{Fc}$ maps in all 10 interfacial active sites in the homodecamer (Figs. 2 and 3 ). The preQ ${ }_{0}$-binding pocket is defined by a cleft between two subunits from the same pentamer. The left wall of the cleft is formed by the loops connecting helices $\alpha 1$ and $a 2$ (Ser54- Leu61), strands $\beta 1$ and $\beta 2$ (Cys21- Thr26), and strands $\beta 3$ and $\beta 4$ (Tyr90- Val93), as well as helix a 2 (Leu61-Asn78) and strand $\beta 4$ (Val93- Gly103), all from one subunit. The right and back walls of the cleft are formed by the N-terminal half of strand $\beta 1$ (Val11- Arg16), the N-terminal half of helix a 1 (Ala44Lys49), and an extended N-terminal polypeptide stretch from the adjacent subunit (Val4Ser9). A covalent bond is formed between the sulfur atom of the conserved active site Cys21 and the nitrile carbon of the $\mathrm{preQ}_{0}$ (Fig. 3). Similar to QueF, the $\mathrm{preQ}_{0}$ binding pocket in QueF-L is formed by interface residues from both subunits (Fig. 3). From one subunit, a Glu63 side chain forms hydrogen bonds with the $\mathrm{N} 1$ and $\mathrm{N} 2$ atoms of $\mathrm{preQ}_{0}$, and the backbone amine of His62 forms a hydrogen bond with $\mathrm{O} 6$ of $\mathrm{preQ}_{0}$. From the same subunit, the Asp28 side chain makes a hydrogen bond with the thioimide nitrogen. From the adjacent subunit, the side chain oxygen atom of Glu46 and the backbone carbonyl oxygen of Leu43 make hydrogen bonds with $\mathrm{N} 9$ and $\mathrm{N} 2$ of $\mathrm{preQ}_{0}$, respectively. The guanidine ring of $\mathrm{preQ}_{0}$ is sandwiched between two hydrophobic patches; one patch is formed by Ile23 and Leu61 from one subunit, and Leu2 from the neighboring subunit. The second patch includes Tyr90 from one subunit and Ile45 from the neighboring subunit. Overall, the thioimide intermediate formed by QueF-L, and the entire $\mathrm{preQ}_{0}$ binding pocket is very similar to that seen in QueF.

Three-dimensional superposition of the apo enzyme structure with the thioimide intermediate reveals substrate-induced conformational changes in the active site region (Fig. 3 ). In the apo enzyme, the five $\mathrm{N}$-terminal residues are not visible in the electron density map due to disorder. These residues become ordered upon $\mathrm{preQ}_{0}$ binding and form an extended tail that packs against the $\mathrm{preQ}_{0}$-occupied active site (Fig. 3). This is accompanied by conformational changes in the side chains of the $\mathrm{preQ}_{0}$ binding residues Cys21, Asp28, His62, Glu63, Leu43, and Glu46 and narrowing of the active site cavity in an induced-fit fashion. 


\section{The structural basis of the different catalytic activities of QueF and QueF-L}

The QueF enzymes fall into two subfamilies: the unimodular subfamily, exemplified by

Bacillus subtilis QueF, consist of a single T-fold domain with an invariant active site Cys and a "QueF motif" embedded in a helix flanking the active site ${ }^{24}$ (Fig. 4A). The QueF motif $\mathrm{E}(\mathrm{S} / \mathrm{L}) \mathrm{K}(\mathrm{S} / \mathrm{A}) \mathrm{hK}(\mathrm{L} / \mathrm{Y})(\mathrm{Y} / \mathrm{F} / \mathrm{W}$ (where $\mathrm{h}$ is a hydrophobic residue) is characteristic of QueF enzymes and contains residues consistent with NADPH binding. ${ }^{16,24}$ The bimodular subfamily, represented by Vibrio cholerae QueF, consists of two weakly homologous tandem T-fold domains in which the QueF motif and the active site Cys residue are separated in the two domains and join together in the tertiary structure to form an inter-domain active site (Figs. 4A and 4B). The unimodular QueF enzymes exist as homodecamers with intersubunit active sites ${ }^{24}$ whereas the bimodular enzymes are homodimers with intra-subunit active $\operatorname{sites}^{25}$ (Fig. 4B).

Based on 3D superposition of $P$. calidifontis QueF-L and representatives of the two QueF subfamilies, B. subtilis QueF (PDB ID 4F8B) and $V$. cholerae QueF (PDB ID 3UXJ), a structure-based multisequence alignment was generated (Fig. 4C). The alignment shows that QueF-L enzymes are most similar to the unimodular QueF (e.g., $P$. calidifontis QueF-L and B. subtilis QueF exhibit $19 \%$ sequence identity and superpose with r.m.s.d. $1.4 \AA$ over $99 \mathrm{C}_{\mathrm{a}}$ atoms) except that they lack the 10 -residue $\mathrm{N}$-terminal $\beta$-strand and 30 -residue $\mathrm{C}$-terminal a-helix of QueF. They are also similar to the C-terminal domains of bimodular QueF (e.g. P. calidifontis QueF-L and V. cholerae QueF share 18\% sequence identity and superpose with r.m.s.d. $1.4 \AA$ over $94 \mathrm{C}_{\alpha}$ atoms). The eight common residues between QueF-L and QueF are the five active site residues (Cys21, Asp28, Tyr36, and Glu63, in P. calidifontis residue numbers) and three structural residues (Ser54, Val84, and Gly92).

Most significantly, the QueF motif in helix a1 (e.g. $\mathrm{E}_{78} \mathrm{SKShKL}_{84}$ in B. subtilis QueF) is not conserved in QueF-L (Fig. 4C). The QueF motif is involved in NADPH binding by QueF enzymes, as revealed by the crystal structure of $V$. cholerae QueF in complex with NADP where the bound NADP molecule spans the inter-subunit interface between two $\mathrm{preQ}_{0}$ binding sites (Fig. 5A, the Midwest Center for Structural Genomics, PDB ID 3UXJ). Instead of the QueF motif, the a 1 helix in QueF-L harbors on its hydrophilic face 4 conserved ionic side chains (Glu18, Lys49, Asp53, Arg56 in P. calidifontis QueF-L) that are seen in the crystal structure fully engaged in inter-pentamer salt bridge interactions that pin together the two pentameric subunits (Fig. 5C). In contrast, the two pentamers in unimodular QueF are held together by conserved inter-pentamer cationic metal sites and salt bridges formed by residues in the C-terminal a3-helix-tails (Fig. 5B, in B. subtilis QueF these residues are Asp162 and Asp131-Arg164 for the metal site and salt bridge, respectively). ${ }^{24}$ These extended, rigid C-terminal tails act as spacers between the QueF pentamers, thus creating a pocket at the inter-pentamer interface for NADPH binding. In QueF-L, which lacks the Cterminal helix-tail spacers, the two pentamers pack closely against each other via salt bridges, leaving no space at the interface for a binding site suitable for a large secondary ligand such as NADPH (Fig. 5C). These differences result in a 19-degree clockwise screw rotation around the tunnel axis of the top pentamer relative to the bottom pentamer in QueFL, as compared to the relatively "unscrewed" and looser pentamers of unimodular QueF. 
In summary, although the quaternary structures of unimodular QueF and QueF-L are similar (both C5-symmetric homodecamers), the QueF-L homodecamer is much more tightly "screwed" together (Fig. 4) due to the minimal T-fold tertiary structure of the monomer. Similarly, in the bimodular QueF dimer, the monomers are aligned and farther apart, allowing space for NADPH binding at the interface (Fig. 5A). Because QueF enzymes are more ancient (e.g. QueF is found in the ancient bacterium Aquifex aeolicus which branches close to the root of the phylogenetic tree of life), these structural differences highlight the path of molecular evolution from the more ancient QueF enzymes catalyzing NADPHdependent nitrile reduction to the more recent QueF-L enzymes catalyzing amidino transfer.

\section{Putative tRNA binding sites}

Biochemical characterization of recombinant QueF-L demonstrated that while QueF-L binds to free $\mathrm{pre}_{0}$ and forms a covalent thioimide intermediate analogous to QueF, only pre $\mathrm{Q}_{0^{-}}$ tRNA serves as substrate for turnover, producing $\mathrm{G}^{+}$-tRNA (D. Iwata-Reuyl, manuscript in preparation). This makes QueF-L the only example of a T-fold enzyme that binds a nucleic acid. Indeed, QueF-L displays a large positively charged outer surfaces on both flat faces of the decamer that could provide sites for recognition of the tRNA phosphate backbone (Fig. $6)$.

In the crystal structure of ArcTGT bound to tRNA (P. horikoshii ArcTGT and tRNA Val, PDB ID $\left.1 \mathrm{~J}_{2} \mathrm{~B}^{39}\right)$, ArcTGT drastically alters the conformation of tRNA to adopt a noncanonical form, the $\lambda$ form, in which the secondary base pairs and canonical tertiary interactions are disrupted and the D-arm of tRNA is mostly single stranded and protruded, and the D-loop in an extended conformation. ArcTGT precisely locates the exposed G15 base in the active site by counting the nucleotides from G1 to G15 along the D-arm.

To predict the putative tRNA binding site on QueF-L, we docked the D-loop of the $\lambda$ form of P. horikoshii tRNA ${ }^{\mathrm{Val}}$ as seen in the crystal structure of its complex with ArcTGT (PDB ID $1 \mathrm{~J}_{2} \mathrm{~B}^{39}$ ) using the HADDOCK webserver. ${ }^{38}$ HADDOCK (High Ambiguity Driven biomolecular DOCKing) is a data driven method that predicts minimal energy conformations from experimentally or bioinformatically available interaction information. Attempts to dock the D-loop from the L-form of the same tRNA (PDB ID 4TNA) failed due to inaccessibility of the $\mathrm{D}$ loop, indicating that the extended conformation of the $\lambda$-form $\mathrm{D}$ stem-loop is the more likely form recognized by QueF-L. The resulting energy-minimized model shows the $\lambda$-form D-loop in an extended conformation bound to a positively charged surface groove that extends across the inter-subunit interface and over the active site (Fig. 6). The RNA phosphate backbone lays on the surface whereas the nucleobase at position 15 is buried deeply within the $\mathrm{pre}_{0}$ binding pocket. The model also shows that the bases of Dloop residues before and after the modification site (A14 and C16 in P. horikoshii RNA $^{\mathrm{Val}}$ ) point away into solvent and do not interact with the protein, suggesting that QueF-L recognizes the D-loop in a sequence-independent manner. Indeed, $P$. calidifontis QueF-L was shown to be able to modify the $\mathrm{preQ}_{0}$ base at position 34 (the wobble position) in the anticodon loop of bacterial tRNA ${ }^{\text {Asp }}$, which bears a different sequence context from the $\mathrm{D}$ loop of the natural QueF-L substrate. (Specifically, tRNA ${ }^{\text {Asp }}$ isolated from an engineered $\triangle q u e F E$. coli mutant strain expressing P. calidifontis QueF-L was found to contain $\mathrm{G}^{+}$at 
position 34, where the promiscuous bacterial $\mathrm{TGT}^{40}$ inserted $\mathrm{preQ}_{0}$ instead of the unavailable pre $\mathrm{Q}_{1}{ }^{30}$ ). These findings are consistent with our model in which RNA recognition by QueF-L is structure-specific rather than sequence-specific.

\section{Putative ammonia pocket and gate}

Although the present structures do not contain any bound ammonium ions or ammonia, they provide insight into the possible mechanism of ammonia delivery to the active sites. Near all active sites, a solvent filled cavity, $79 \AA^{3}$ in volume and formed by Tyr89 and Tyr90 and their symmetry mates from the opposite pentamer, poses as a putative pocket for the binding of ammonium ions (Fig. 7) through cation- $\pi$ interactions with the Tyr90 side chains. ${ }^{41}$ This cavity is at the inter-pentamer interface and is bordered on one side by bound $\mathrm{preQ}_{0}$, Asp28 and the two symmetry-related Tyr90 side chains, and on the other side by the two Tyr89 side chains which separate the cavity from the central tunnel of the enzyme decamer. It is possible for the Tyr89 side chain to adopt an alternate conformation that opens the cavity to the central tunnel of the enzyme, much like a "gate" (Fig. 7), allowing flow of ammonium ions from the central tunnel to the active site via the cavity, similar to the ammonia channel known for other amidotransferases (see below).

\section{Proposed mechanism for thioimide formation and amidino transfer}

QueF and QueF-L bind and react with pre $\mathrm{Q}_{0}$ and $\mathrm{preQ}_{0}$-tRNA to form a covalent thioimide intermediate, but QueF reduces the thioimide intermediate to a primary amine, while QueF$\mathrm{L}$ delivers $\mathrm{NH}_{3}$ to the thioimide intermediate to form a formamidino group. The chemical divergence is a consequence of the binding of secondary ligands, NADPH for QueF and $\mathrm{NH}_{4}+$ for QueF-L. However, the conservation of all the thioimide interactions indicates that the two enzymes likely share the same mechanism for thioimide formation.

Based on the structures reported here and the similarities noted above to the structure of $B$. subtilis QueF, we propose that binding of $\mathrm{preQ}_{0}$-tRNA is facilitated by $\mathrm{H}$-bonding interactions with Glu63 and Glu46 (Fig. 3). Glu63 helps to position the substrate in the active site through $\mathrm{H}$-bonding with $\mathrm{N} 1$ and $\mathrm{N} 2$ (a conserved interaction of all T-fold proteins), while Glu46 serves as a proton donor to $\mathrm{N} 9$ of preQ $\mathrm{Q}_{0}$-tRNA such that the lone-pair is unable to delocalize into the $\pi$-system defined by the nitrile and the double bond of the deazaguanine ring. This has the effect of increasing the reactivity (electrophilicity) of the nitrile carbon (and subsequently the thioimide carbon) to nucleophilic attack. Asp28 deprotonates Cys21, enabling it to nucleophilically attack the nitrile carbon of preQ $_{0}$ to produce the covalent thioimide intermediate (Fig. 8).

The ammonium cation, binding in the pocket defined by Asp28 and the pi-system of Tyr90, is deprotonated by Asp28, allowing the neutral ammonia to attack the thioimide carbon from the Tyr90 side (the face of $\mathrm{preQ}_{0}$ facing the protein core). Proton transfers appear to be facilitated in this process via a tightly bound water molecule observed in the $\mathrm{preQ}_{0}$-bound structure that is H-bonded to His62 and the thioimide nitrogen atom. Collapse of the resulting diaminothioorthoester intermediate via cleavage of the carbon-sulfur bond then provides archaeosine-modified tRNA. 
The failure of QueF-L to turnover the thioimide intermediate formed from $\mathrm{proQ}_{0}$ appears to be due to the structural consequences of $\mathrm{pre}_{0}$ binding; as noted above, $\mathrm{pre}_{0}$ binding induces the five $\mathrm{N}$-terminal residues of the adjacent subunit harboring Glu46 to form an

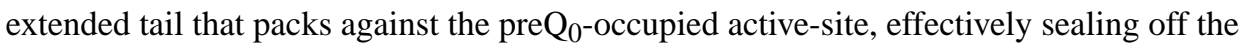
active-site from the bulk solvent and locking the structure. This structural rearrangement presumably cannot occur when preQ $_{0}$-modified tRNA binds, as the ribosyl moiety and the extended RNA preclude such packing. Furthermore, these structural changes appear to preclude opening of the putative Tyr90 "gate" for ammonium ions to access the active site from the central tunnel.

\section{New chemistry for a common fold and new fold for a common chemistry}

The amidinotransfer reaction catalyzed by QueF-L is the seventh reaction now shown to be supported by the T-fold. The other six reactions are cofactor independent oxidations by the urate and coproporphyrinogen III oxidases, ${ }^{42}$ hydrolysis and rearrangement by the GTP cyclohydrolase I enzymes (GCYH-IA and IB), carbon-carbon bond cleavage by dihydroneopterin adolase (DHNA) ${ }^{43}$ and 6-carboxytetrahydropterin synthase, ${ }^{14}$ isomerization and elimination reactions by 6-pyruvoyl tetrahydropterin synthase (PTPS) ${ }^{44}$ and 7,8-dihydroneopterin triphosphate epimerase, ${ }^{45}$ and NADPH-dependent reduction catalyzed by QueF. ${ }^{16}$

Amidotransfer reactions are catalyzed by a number of enzyme families that generate ammonia differently and act on different substrates. The most characterized are the glutamine-dependent amidotransferases (GATase) which catalyze amidation of a variety of small molecule substrates and utilize glutamine as an ammonia donor. ${ }^{46}$ They harbor a conserved glutaminase domain for ammonia production and a variable receptor domain for product synthesis. These enzymes fall into two classes based on the architecture of the glutaminase domain and the mechanism of ammonia production. The Class-I GATases (also known as the Triad class, e.g. carbamoyl phosphate synthetase) utilize a conserved glutaminase domain with the characteristic catalytic triad Cys/His/Glu to hydrolyze glutamine and generate ammonia. The glutaminase domain in this class adopts a conserved $a \beta a$ flavodoxin-like fold constituted of a 5-stranded parallel $\beta$-sheet and flanking helices. The Class II enzymes (also known as purF-type, Ntn or N-terminal nucleophile class, e.g. glucosamine 6 phosphate synthase) follow a $\alpha \beta \beta a$ fold where two extended largely antiparallel $\beta$ sheets face each other and are flanked by layers of $\alpha$ helices. This class employs an essential $\mathrm{N}$-terminal cysteine residue to generate ammonia from glutamine. Some members from either class are ATP dependent and activate substrates by phosphorylation or adenylation prior to amidation. In both classes, the ammonia is transported through a channel that runs through the core of the protein from the glutaminase active site to the amidation site in the receptor domain.

More recently, tRNA-dependent amidotransferases that recognize and act on tRNA substrates were identified and characterized. These include the heterotrimeric GatCAB enzymes which convert Glu-tRNA ${ }^{\text {Gln }} /$ Asp-tRNA ${ }^{\text {Asn }}$ to Gln-tRNA ${ }^{\text {Gln }} /$ Asn-tRNA ${ }^{\text {Asn }}$ in some bacteria and archaea and utilize Gln or Asn as ammonia sources, ${ }^{47}$ and the heterodimeric GatDE enzymes which convert Glu-tRNA ${ }^{\text {Gln }}$ to Gln-tRNA ${ }^{\text {Gln }}$ in archaea and use only Gln as 
an ammonia source. ${ }^{48}$ Both enzyme families are ATP dependent and operate by phosphorylating the gamma carboxyl group of the Glu/Asp residue attached to tRNA prior to amidation. These enzymes utilize distinct folds for production of ammonia, but a common cradle fold for binding and activation of substrate aminoacyl-tRNAs. Specifically, the ammonia-producing subunit in GatCAB, subunit $\mathrm{A}$, is a single-domain amidase-fold protein consisting of a central, mixed 11-stranded $\beta$-sheet core covered on both sides by a-helices, ${ }^{49}$ whereas the ammonia-producing subunit in GatDE, subunit $\mathrm{D}$, is a multi-domain protein harboring a Class II (Ntn type) glutaminase domain. ${ }^{48,50}$ The respective receptor subunits B and E, where aminoacyl-tRNA is activated, are structurally similar and are built around a unique cradle-shaped core domain made of a twisted 10-stranded mixed $\beta$-sheet packed on its convex side with three a-helices. ${ }^{48-50}$ Despite the variety of folds utilized by the amidotransferase families, they all share the common feature of a 20-40 A long channel in which ammonia is transported from the ammonia production site to the amidation site.

QueF-L and ArcS are structurally unrelated to all of the class I and II and tRNA-dependent amidotransferases (and to each other), representing alternative solutions to the problem of delivering ammonia to an activated substrate. Furthermore, QueF-L is the first such enzyme that relies exclusively on free ammonia instead of glutamine or asparagine, although we cannot rule out that in vivo QueF-L might function with a partner that serves to hydrolyze glutamine/asparagine. Although it has been established that ArcS and QueF-L convert a nitrile group to a formamidine, they remain the least characterized of the amidotransferase families.

\section{CONCLUSION}

QueF and QueF-L act on similar substrates ( $\mathrm{preQ}_{0}$ and $\mathrm{preQ}_{0}$-tRNA), but catalyze different chemistries. They accommodate the $\mathrm{pre}_{0}$ moiety in strikingly similar active sites, but they produce different products. This is reminiscent of the case of mPTPS and QueD, two other enzymes in the 7-deazaguanosine pathway that harbor similar active sites and bind the same substrate (7,8-dihydroneopterin triphosphate) but generate different products; 6pyruvoyltetrahydropterin and 6-carboxy-5,6,7,8-tetrahydropterin, respectively). ${ }^{23}$ The structural basis of the difference in catalytic function between QueF and QueF-L can be attributed to subtle differences in the enzyme quaternary assembly caused by structural elements outside the T-fold, giving rise to different binding sites for secondary ligands (NADPH versus $\mathrm{NH}_{4}+$ ). This work highlights how minor changes in a highly conserved fold have led to the emergence of different catalytic functions.

\section{Acknowledgments}

We thank Ms. Adeba Mohammad and Shivani Thacker for providing general lab support during this project. The Stanford Synchrotron Research Laboratory Structural Molecular Biology Program is supported by the National Institutes of Health. This project is supported by NSF grant CHE- 1309323 to D. Iwata-Reuyl and M.A. Swairjo.

\section{References}

1. Kasai H, Ohashi Z, Harada F, Nishimura S, Oppenheimer NJ, Crain PF, Liehr JG, von Minden DL, McCloskey JA. Structure of the modified nucleoside Q isolated from Escherichia coli transfer 
ribonucleic acid. 7-(4,5-cis-Dihydroxy-1-cyclopenten-3-ylaminomethyl)-7-deazaguanosine. Biochemistry. 1975; 14(19):4198-4208. [PubMed: 1101947]

2. Ohgi T, Kondo T, Goto T. Total synthesis of optically pure nucleoside Q. Determination of absolute configuration of natural nucleoside Q. J Am Chem Soc. 1979; 101:3629-3633.

3. Okada N, Nishimura S. Enzymatic synthesis of Q* nucleoside containing mannose in the anticodon of tRNA: isolation of a novel mannosyltransferase from a cell-free extract of rat liver. Nucleic Acids Res. 1977; 4(8):2931-2937. [PubMed: 20603]

4. Campanacci V, Dubois DY, Becker HD, Kern D, Spinelli S, Valencia C, Pagot F, Salomoni A, Grisel S, Vincentelli R, Bignon C, Lapointe J, Giege R, Cambillau C. The Escherichia coli YadB gene product reveals a novel aminoacyl-tRNA synthetase like activity. J Mol Biol. 2004; 337(2):273-283. [PubMed: 15003446]

5. Salazar JC, Ambrogelly A, Crain PF, McCloskey JA, Soll D. A truncated aminoacyl-tRNA synthetase modifies RNA. Proc Natl Acad Sci U S A. 2004; 101(20):7536-7541. [PubMed: 15096612]

6. Gregson JM, Crain PF, Edmonds CG, Gupta R, Hashizume T, Phillipson DW, McCloskey JA. Structure of archaeal transfer RNA nucleoside G*-15 (2-Amino-4,7-dihydro-4-oxo-7-b-Dribofuranosyl-1H-pyrrolo[2,3-d]pyrimidine-5-carboximidamide (Archaeosine)). J Biol Chem. 1993; 268(14):10076-10086. [PubMed: 7683667]

7. Kersten $H$. The nutrient factor queuine: biosynthesis, occurence in transfer RNA and function. Biofactors. 1988; 1(1):27-29. [PubMed: 3076430]

8. Sprinzl M, Vassilenko KS. Compilation of tRNA sequences and sequences of tRNA genes. Nucleic Acids Res. 2005; 33(Database issue):D139-D140. [PubMed: 15608164]

9. Meier F, Suter B, Grosjean H, Keith G, Kubli E. Queuosine modification of the wobble base in tRNA $^{\text {His }}$ influences 'in vivo' decoding properties. EMBO J. 1985; 4(3):823-827. [PubMed: 2988936]

10. Carlson BA, Kwon SY, Chamorro M, Oroszlan S, Hatfield DL, Lee BJ. Transfer RNA modification status influences retroviral ribosomal frameshifting. Virology. 1999; 255(1):2-8. [PubMed: 10049815]

11. Oliva R, Tramontano A, Cavallo L. $\mathrm{Mg}^{2+}$ binding and archaeosine modification stabilize the G15 C48 Levitt base pair in tRNAs. RNA. 2007; 13(9):1427-1436. [PubMed: 17652139]

12. Phillips G, El Yacoubi B, Lyons B, Alvarez S, Iwata-Reuyl D, de Crecy-Lagard V. Biosynthesis of 7-deazaguanosine-modified tRNA nucleosides: a new role for GTP cyclohydrolase I. J Bacteriol. 2008; 190:7876-7884. [PubMed: 18931107]

13. Reader J, Metzgar D, Schimmel P, de Crécy-lagard V. Identification of four genes necessary for biosynthesis of the modified nucleoside queuosine. J Biol Chem. 2004; 279:6280-6285. [PubMed: 14660578]

14. McCarty RM, Somogyi A, Lin G, Jacobsen NE, Bandarian V. The deazapurine biosynthetic pathway revealed: in vitro enzymatic synthesis of $\operatorname{PreQ}(0)$ from guanosine 5'-triphosphate in four steps. Biochemistry. 2009; 48(18):3847-3852. [PubMed: 19354300]

15. Blaby IK, Phillips G, Blaby-Haas CE, Gulig KS, El Yacoubi B, de Crecy-Lagard V. Towards a systems approach in the genetic analysis of archaea: Accelerating mutant construction and phenotypic analysis in Haloferax volcanii. Archaea. 2010; 2010:426239. [PubMed: 21234384]

16. Van Lanen SG, Reader JS, Swairjo MA, de Crecy-Lagard V, Lee B, Iwata-Reuyl D. From cyclohydrolase to oxidoreductase: discovery of nitrile reductase activity in a common fold. Proc Natl Acad Sci U S A. 2005; 102(12):4264-4269. [PubMed: 15767583]

17. Noguchi S, Nishimura Y, Hirota Y, Nishimura S. Isolation and characterization of an Escherichia coli mutant lacking tRNA-guanine-transglycosylase. Function and biosynthesis of queuosine in tRNA. J Biol Chem. 1982; 257(11):6544-6550. [PubMed: 6804468]

18. Watanabe M, Matsuo M, Tanaka S, Akimoto H, Asahi S, Nishimura S, Katz JR, Hashizume T, Crain PF, McCloskey JA, Okada N. Biosynthesis of archaeosine, a novel derivative of 7deazaguanosine specific to archaeal tRNA, proceeds via a pathway involving base replacement of the tRNA polynucleotide chain. J Biol Chem. 1997; 272(32):20146-20151. [PubMed: 9242689]

19. Bai Y, Fox DT, Lacy JA, Van Lanen SG, Iwata-Reuyl D. Hypermodification of tRNA in thermophilic archaea. Cloning, overexpression, and characterization of tRNA-guanine 
transglycosylase from Methanococcus jannaschii. J Biol Chem. 2000; 275(37):28731-28738. [PubMed: 10862614]

20. Phillips G, Chikwana VM, Maxwell A, El-Yacoubi B, Swairjo MA, Iwata-Reuyl D, de CrécyLagard V. Discovery and characterization of an amidotransferase involved in the modification of archaeal tRNA. J Biol Chem. 2010; 285(17):12706-12713. [PubMed: 20129918]

21. Nar H, Huber R, Meining W, Schmid C, Weinkauf S, Bacher A. Atomic structure of GTP cyclohydrolase. Structure (Camb). 1995; 3(5):459-466. [PubMed: 7663943]

22. Sankaran B, Bonnett SA, Shah K, Gabriel S, Reddy R, Schimmel P, Rodionov DA, de CrecyLagard V, Helmann JD, Iwata-Reuyl D, Swairjo MA. Zinc-independent folate biosynthesis: genetic, biochemical, and structural investigations reveal new metal dependence for GTP cyclohydrolase IB. J Bacteriol. 2009; 191(22):6936-6949. [PubMed: 19767425]

23. Miles ZD, Roberts SA, McCarty RM, Bandarian V. Biochemical and structural studies of 6carboxy-5,6,7,8-tetrahydropterin synthase reveal the molecular basis of catalytic promiscuity within the tunnel-fold superfamily. J Biol Chem. 2014; 289(34):23641-23652. [PubMed: 24990950]

24. Chikwana VM, Stec B, Lee BW, de Crecy-Lagard V, Iwata-Reuyl D, Swairjo MA. Structural basis of biological nitrile reduction. J Biol Chem. 2012; 287(36):30560-30570. [PubMed: 22787148]

25. Kim Y, Zhou M, Moy S, Morales J, Cunningham MA, Joachimiak A. High-resolution structure of the nitrile reductase QueF combined with molecular simulations provide insight into enzyme mechanism. J Mol Biol. 2010; 404:127-137. [PubMed: 20875425]

26. Colloc'h N, Poupon A, Mornon J-P. Sequence and structural features of the T-fold, an original tunneling building unit. Proteins. 2000; 39:142-154. [PubMed: 10737935]

27. Green, JC.; Nichols, BP.; Mathhews, RG. Folate biosynthesis, reduction, and polyglutamylation. In: Neidhart, FC., editor. Escherichia coli and Salmonella, Cellular and Molecular Biology. Washington, DC: American Society for Microbiology; 1996. p. 665-673.

28. Thony B, Auerbach G, Blau N. Tetrahydrobiopterin biosynthesis, regeneration and functions. Biochem J. 2000; 347(Pt 1):1-16. [PubMed: 10727395]

29. McCarty RM, Somogyi A, Bandarian V. Escherichia coli QueD Is a 6-carboxy-5,6,7,8tetrahydropterin synthase. Biochemistry. 2009; 48(11):2301-2303. [PubMed: 19231875]

30. Phillips G, Swairjo MA, Gaston KW, Bailly M, Limbach PA, Iwata-Reuyl D, de Crécy-Lagard V. Diversity of archaeosine synthesis in crenarchaeota. ACS Chem Biol. 2011; 7:300-305. [PubMed: 22032275]

31. Chacón KN, Blackburn NJ. Stable $\mathrm{Cu}(\mathrm{II})$ and $\mathrm{Cu}(\mathrm{I})$ mononuclear intermediates in the assembly of the CuA center of Thermus thermophilus cytochrome oxidase. J Am Chem Soc. 2012; 134(39): 16401-16412. [PubMed: 22946616]

32. Migawa MT, Hinkley JM, Hoops GC, Townsend LB. A two step synthesis of the nucleoside Q precursor 2-amino-5-cyanopyrrolo[2,3- $d$ ] pyrimidin-4-one $\left(\mathrm{PreQ}_{0}\right)$. Synth Comm. 1996; 26(17): 3317-3322.

33. Otwinowski Z, Minor W. Processing of X-ray diffraction data collected in oscillation mode. Methods Enzymol. 1997; 276:307-326.

34. Adams PD, Afonine PV, Bunkoczi G, Chen VB, Davis IW, Echols N, Headd JJ, Hung LW, Kapral GJ, Grosse-Kunstleve RW, McCoy AJ, Moriarty NW, Oeffner R, Read RJ, Richardson DC, Richardson JS, Terwilliger TC, Zwart PH. PHENIX: a comprehensive Python-based system for macromolecular structure solution. Acta Crystallogr D Biol Crystallogr. 2010; 66(Pt 2):213-221. [PubMed: 20124702]

35. Murshudov GN, Skubak P, Lebedev AA, Pannu NS, Steiner RA, Nicholls RA, Winn MD, Long F, Vagin AA. REFMAC5 for the refinement of macromolecular crystal structures. Acta Crystallogr D Biol Crystallogr. 2011; 67(Pt 4):355-367. [PubMed: 21460454]

36. Emsley P, Lohkamp B, Scott WG, Cowtan K. Features and development of Coot. Acta Crystallogr D Biol Crystallogr. 2010; 66(Pt 4):486-501. [PubMed: 20383002]

37. McCoy AJ, Grosse-Kunstleve RW, Adams PD, Winn MD, Storoni LC, Read RJ. Phaser crystallographic software. J Appl Crystallogr. 2007; 40:658-674. [PubMed: 19461840] 
38. van Zundert GC, Rodrigues JP, Trellet M, Schmitz C, Kastritis PL, Karaca E, Melquiond AS, van Dijk M, de Vries SJ, Bonvin AM. The HADDOCK2. 2 web server: user-friendly integrative modeling of biomolecular complexes. J Mol Biol. 2016; 428(4):720-725. [PubMed: 26410586]

39. Ishitani R, Nureki O, Nameki N, Okada N, Nishimura S, Yokoyama S. Alternative tertiary structure of tRNA for recognition by a posttranscriptional modification enzyme. Cell. 2003; 113(3):383394. [PubMed: 12732145]

40. Hoops GC, Townsend LB, Garcia GA. tRNA-guanine transglycosylase from Escherichia coli: structure-activity studies investigating the role of the aminomethyl substituent of the heterocyclic substrate PreQ1. Biochemistry. 1995; 34(46):15381-15387. [PubMed: 7578154]

41. Dougherty DA. The cation- $\pi$ interaction. Acc Chem Res. 2013; 46(4):885-893. [PubMed: 23214924]

42. Colloc'h N, el Hajji M, Bachet B, L'Hermite G, Schiltz M, Prange T, Castro B, Mornon JP. Crystal structure of the protein drug urate oxidase-inhibitor complex at 2. 05 A resolution. Nat Struct Biol. 1997; 4(11):947-952. [PubMed: 9360612]

43. Hennig M, D'Arcy A, Hampele IC, Page MG, Oefner C, Dale GE. Crystal structure and reaction mechanism of 7,8- dihydroneopterin aldolase from Staphylococcus aureus. Nat Struct Mol Biol. 1998; 5:357-362.

44. Nar H, Huber R, Heizmann CW, Thony B, Burgisser D. Three-dimensional structure of 6-pyruvoyl tetrahydropterin synthase, an enzyme involved in tetrahydrobiopterin biosynthesis. EMBO J. 1994; 13:1255-1262. [PubMed: 8137809]

45. Haussmann C, Rohdich F, Schmidt E, Bacher A, Richter G. Biosynthesis of pteridines in Escherichia coli Structural and mechanistic similarity of dihydroneopterin-triphosphate epimerase and dihydroneopterin aldolase. J Biol Chem. 1998; 273(28):17418-17424. [PubMed: 9651328]

46. Massiere F, Badet-Denisot MA. The mechanism of glutamine-dependent amidotransferases. Cell Mol Life Sci. 1998; 54(3):205-222. [PubMed: 9575335]

47. Tumbula DL, Becker HD, Chang WZ, Soll D. Domain-specific recruitment of amide amino acids for protein synthesis. Nature. 2000; 407(6800):106-110. [PubMed: 10993083]

48. Oshikane H, Sheppard K, Fukai S, Nakamura Y, Ishitani R, Numata T, Sherrer RL, Feng L, Schmitt E, Panvert M, Blanquet S, Mechulam Y, Soll D, Nureki O. Structural basis of RNAdependent recruitment of glutamine to the genetic code. Science. 2006; 312(5782):1950-1954. [PubMed: 16809540]

49. Nakamura A, Yao M, Chimnaronk S, Sakai N, Tanaka I. Ammonia channel couples glutaminase with transamidase reactions in GatCAB. Science. 2006; 312(5782):1954-1958. [PubMed: 16809541]

50. Schmitt E, Panvert M, Blanquet S, Mechulam Y. Structural basis for tRNA-dependent amidotransferase function. Structure. 2005; 13(10):1421-1433. [PubMed: 16216574] 
A

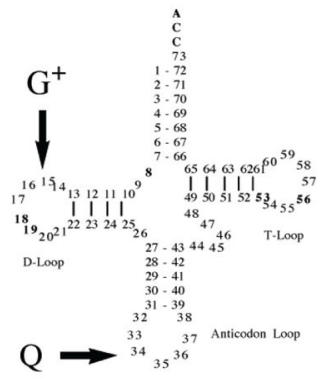

B

Archaea
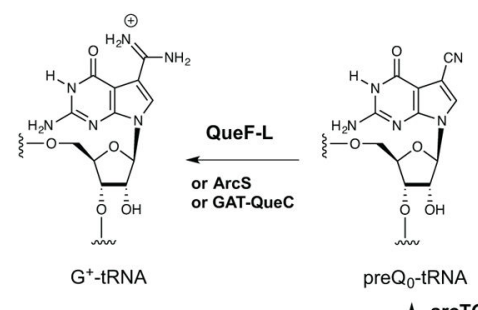

preQ - -tRNA

arcTGT tRNA

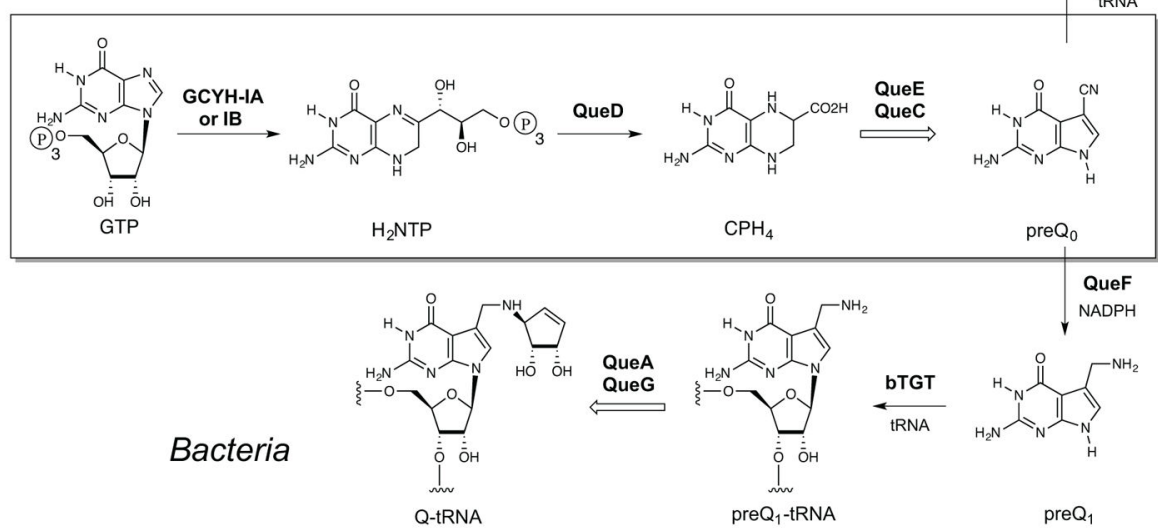

Figure 1.

Biosynthesis of $\mathrm{G}^{+}$. (A) The positions of $\mathrm{Q}$ and $\mathrm{G}^{+}$in bacterial and archaeal tRNA, respectively. (B) The de novo biosynthetic pathway to $\mathrm{Q}$ and $\mathrm{G}^{+}$. The central boxed portion of the pathway is common in Archaea and Bacteria and occurs outside the context of the tRNA and leads to the formation of the modified base 7-cyano-7-deazaguanine ( $\left.\mathrm{preQ}_{0}\right)$. Enzymes that are members of the tunneling-fold superfamily are GCYH-IA and IB, QueD, QueF, and QueF-L. 

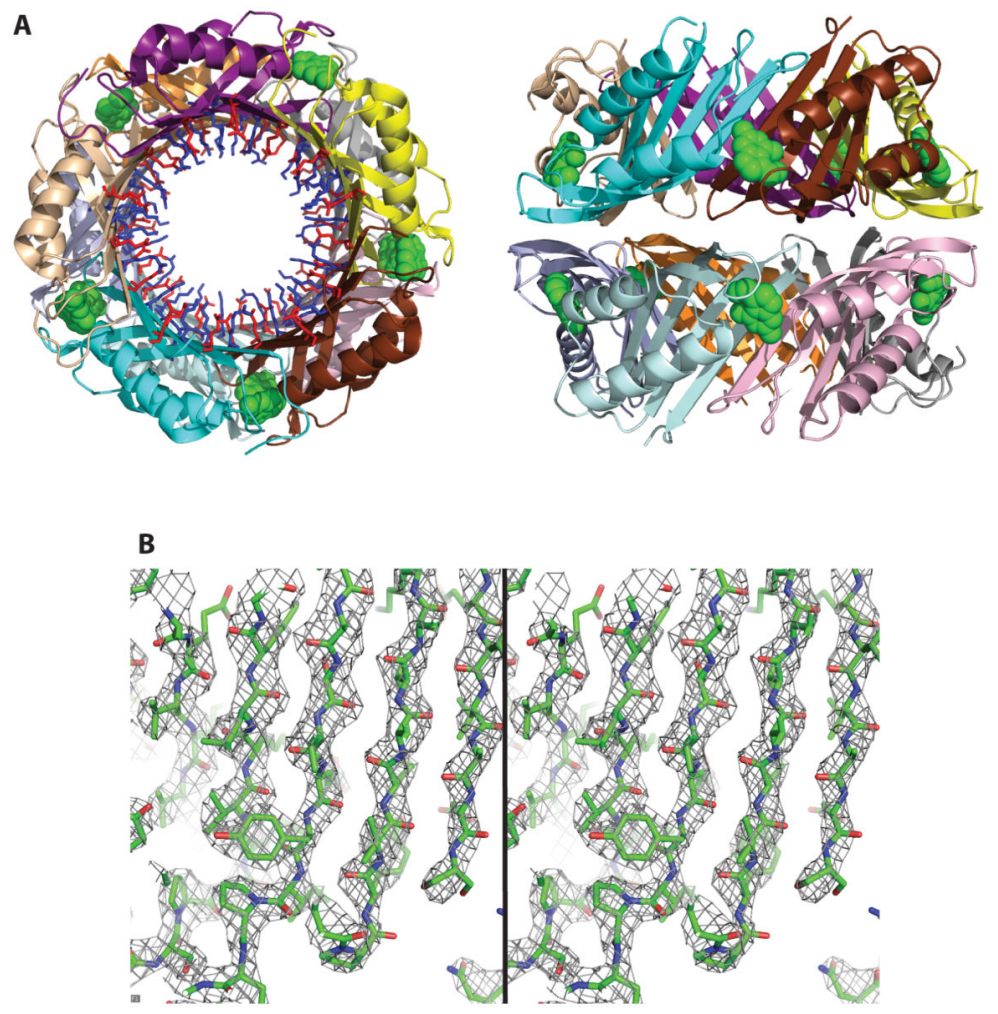

Figure 2.

Crystal structure of QueF-L from P. calidifontis. (A) Ribbon diagram showing a top view (left) and a side view (right) of the QueF-L homodecamer. The monomers are shown in different colors. PreQ $_{0}$ molecules bound in the 10 active sites located at the intersubunit interfaces are shown as green Corey-Pauling-Koltun models. (B) Stereo view of the FOMweighted experimental electron density map (resolution $2.75 \AA$, contour level $1.8 \sigma$ ) calculated after solvent flattening in the $\beta$-sheet region and superimposed on the refined model. 


\section{A}
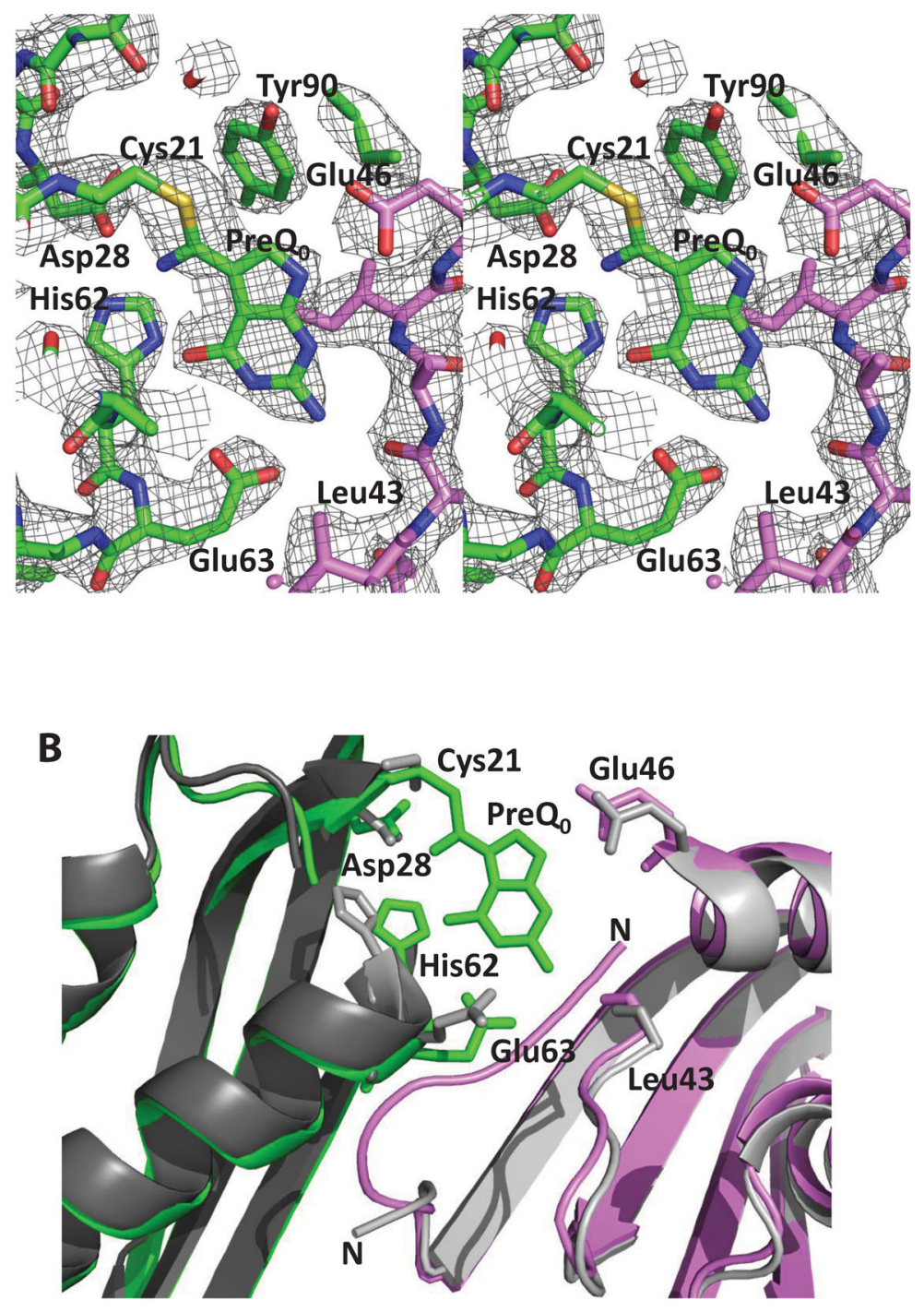

Figure 3.

Formation of a covalent thioimide intermediate in QueF-L and induced-fit binding. (A) Stereo view of the $2 \mathrm{Fo}-\mathrm{Fc}$ electron density map $(2.75 \AA$, contour $1.8 \sigma)$, superposed on the refined model, in one of the active sites showing bound preQ $_{0}$ and formation of thioimide bond with active site Cys21. The carbon atoms in the two interfacial subunits are colored green and pink. (B) Superposition of the apo structure (subunits in dark and light grey) and the preQ $_{0}$-bound structure (subunits in green and pink) around an active site region showing preQ $_{0}$-induced conformational changes. The N-terminus in each structure is indicated. 

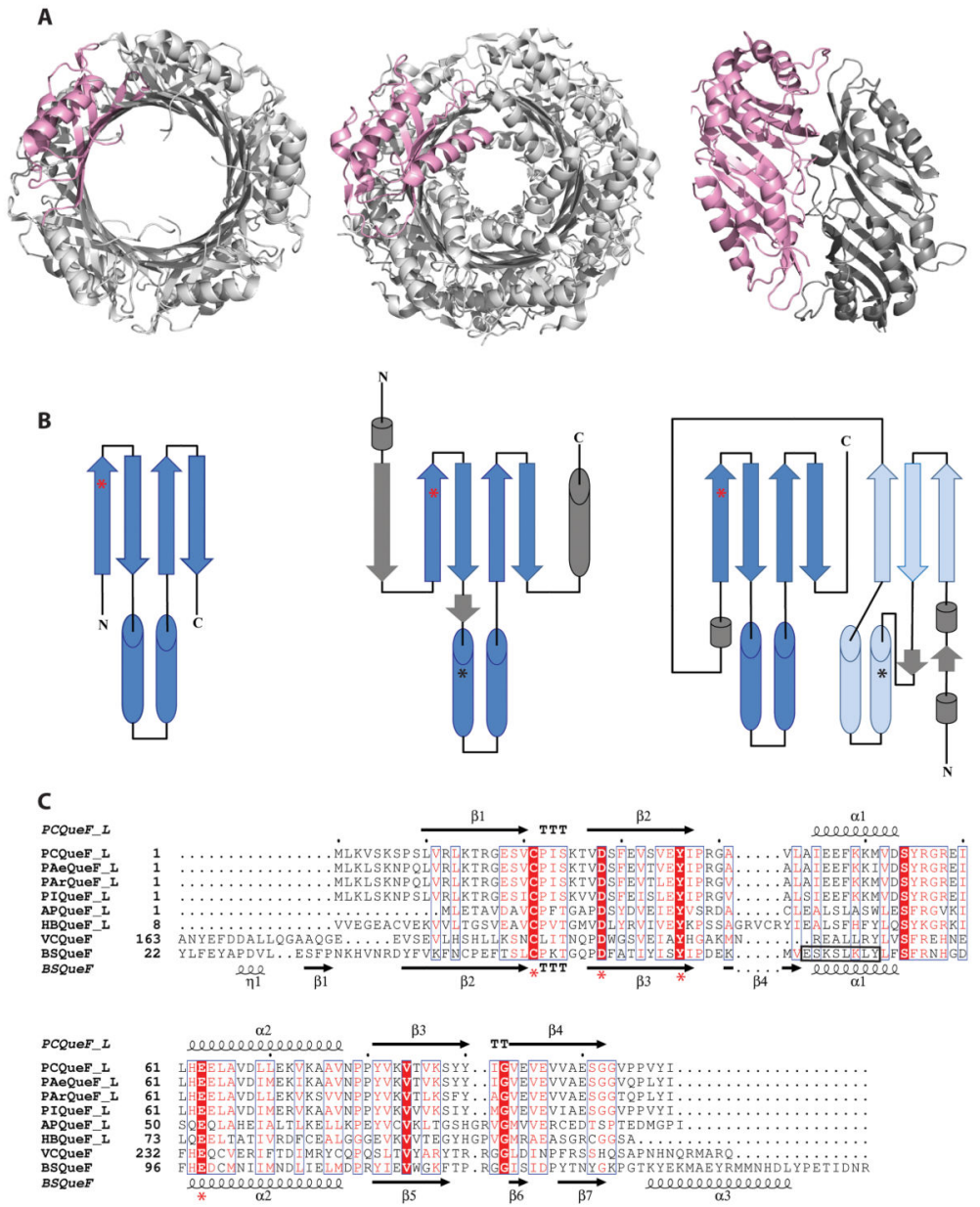

Figure 4.

Structural comparison of QueF-L and QueF enzymes. (A) Ribbon diagrams showing the tertiary and quaternary structures of $P$. calidifontis QueF-L (left), the homodecamer of unimodular QueF from B. subtilis (middle, PDB ID 4F8B), and the homodimer of bimodular QueF from $V$. cholerae (right, PDB ID 3UXJ). Single subunits are colored in pink. (B) Topology diagrams representing the three enzymes, with the common T-fold domain shown in dark blue. $a$-helices and $\beta$-strands are shown as cylinders and arrows, respectively. The incomplete N-terminal T-fold of bimodular QueF is shown in light blue. Locations of the catalytic cysteines and QueF motifs are indicated with red and black asterisks, respectively. (C) Structure-based multi-sequence alignment in the common T-fold region. Secondary structure elements derived from the crystal structures are shown for $P$. calidifontis QueF-L and B. subtilis QueF on top and bottom of the sequence, respectively. Residues shared between the QueF-L and QueF families are highlighted in red background, including residues of the $\mathrm{preQ}_{0}$ binding pocket (red asterisks). The QueF motif is boxed. PC, P. calidifontis; PAe, P. aerophilum; PAr, P. arsenaticum; PI, P. islandicum; AP, A. pernix; HB, H. butylicus; VC, V. cholerae; BS, B. subtilis. 

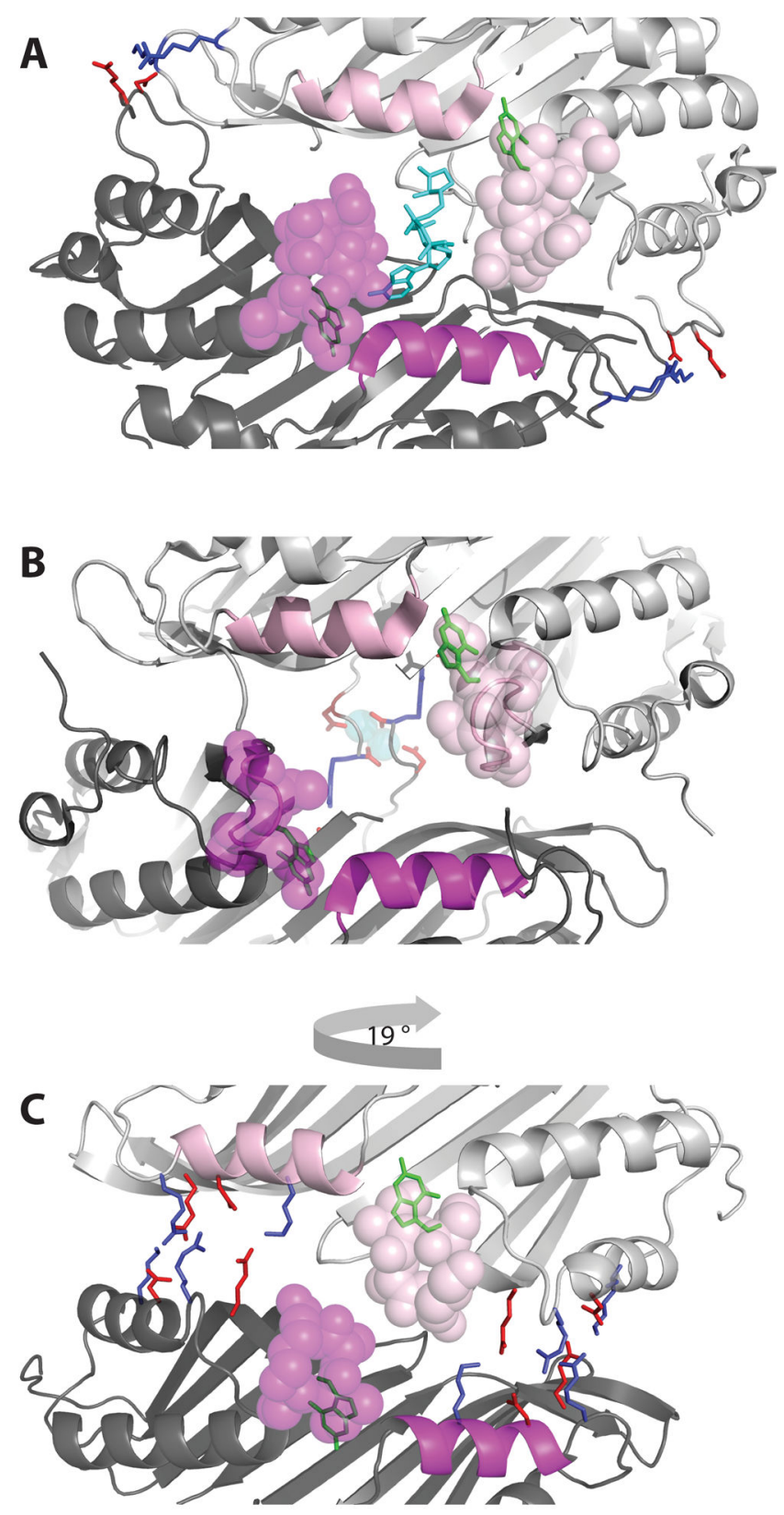

Figure 5.

The QueF-L structure lacks space for NADPH binding. (A) View of the NADPH binding site crystallographically observed at the inter-dimer interface in bimodular QueF ( $V$. cholerae, PDB ID 3UXJ). The two protein subunits are shown as ribbons in different shades of color, and the helices harboring the QueF-motifs are highlighted in purple. Residues of the loops forming the NADPH binding pocket are shown in space-filling model. Bound $\mathrm{preQ}_{0}$ molecules, and the NADPH molecule spanning two preQ $_{0}$-occupied active sites is shown in green and cyan stick model, respectively. (The nicotinamide moiety is missing in this structure due to disorder.) (B) Analogous view and representation of the inter-pentamer interface of unimodular QueF (B. subtilis, PDB ID 4F8B). The C-terminal-tail salt-bridge 
pairs (Asp131-Arg164' and Asp131'-Arg164) and $\mathrm{Mg}^{2+} / \mathrm{Ca}^{2+}$ ion sites that anchor the two pentameric protein subunits together on the tunnel side are shown as blue-red stick model and cyan spheres, respectively. (C) Analogous view and representation of the inter-pentamer interface of $P$. calidifontis QueF-L showing the salt-bridge pairs (Glu18'-Lys49, Asp53Arg56', Asp53'-Arg56, and Glu18-Lys49') that pin together the two pentameric subunits as blue-red sticks. The arrow indicates the relative $19^{\circ}$ inter-pentamer clockwise twist specific to QueF-L. Note the difference between the three enzymes in the relative positions of the a 1 helices across the interface. The average span of the NADPH binding pocket (measured as the inter-loop distance between the closest atoms in the loops averaged over all active sites in the biological multimer) in V. cholerae QueF, B. subtilis QueF, and the analogous distance in QueF-L is $11 \AA, 10 \AA$ and $4 \AA$, respectively. 

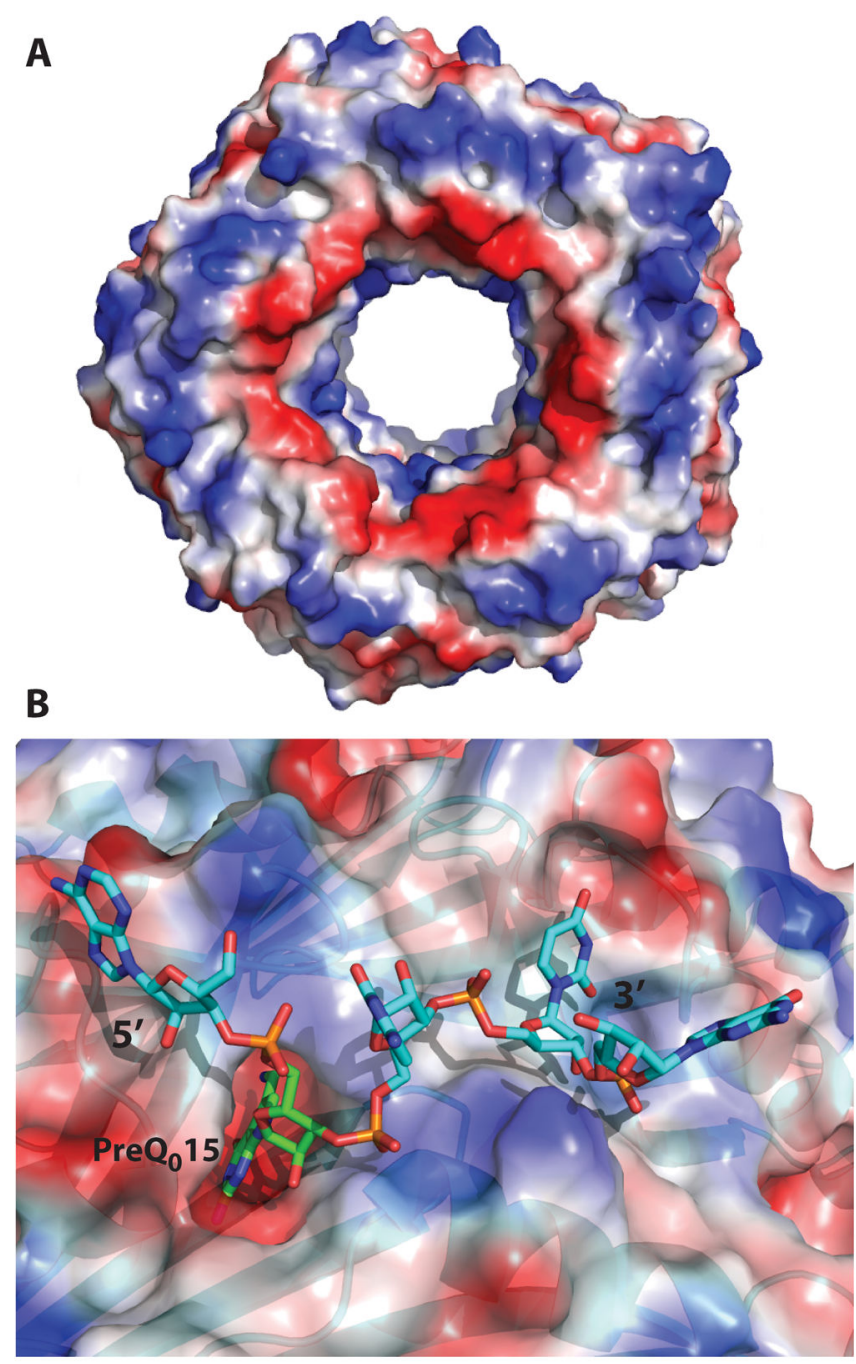

Figure 6.

Putative tRNA binding sites. (A) Surface electrostatic potential of $P$. calidifontis QueF-L. Positive and negative potentials are shown in blue and red, respectively. (B) Energy minimized docking model of a tRNA D-loop containing preQ $\mathrm{Q}_{0}$ at position 15 (ball-and-stick) onto the crystal structure of $P$. calidifontis QueF-L (ribbon and surface rendering). The Dloop phosphate backbone (orange) is recognized by the positively charged groove on the protein surface. The base of G15 is buried deeply in the preQ $_{0}$ binding pocket. 


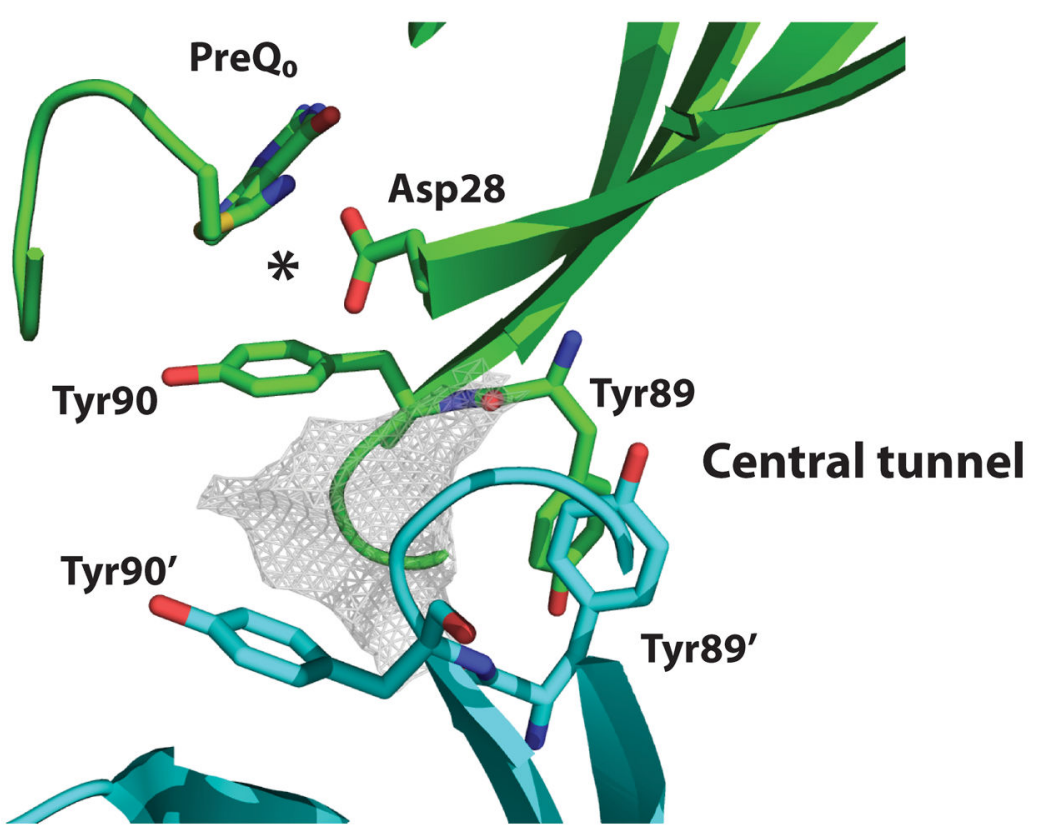

Figure 7.

Putative ammonium channel. View of the inter-pentamer interface near an active site from the preQ $_{0}$-bound QueF-L structure. The putative binding cavity is shown as grey mesh and the enclosing tyrosine side chains are labeled. The asterisk indicates the putative binding site of $\mathrm{NH}^{+}$before amidino transfer. 


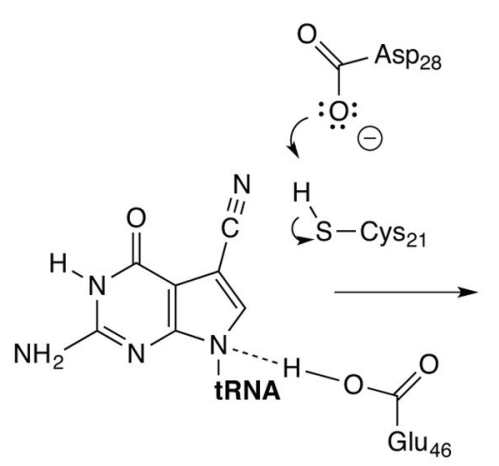

preQ $Q_{0}$-modified tRNA
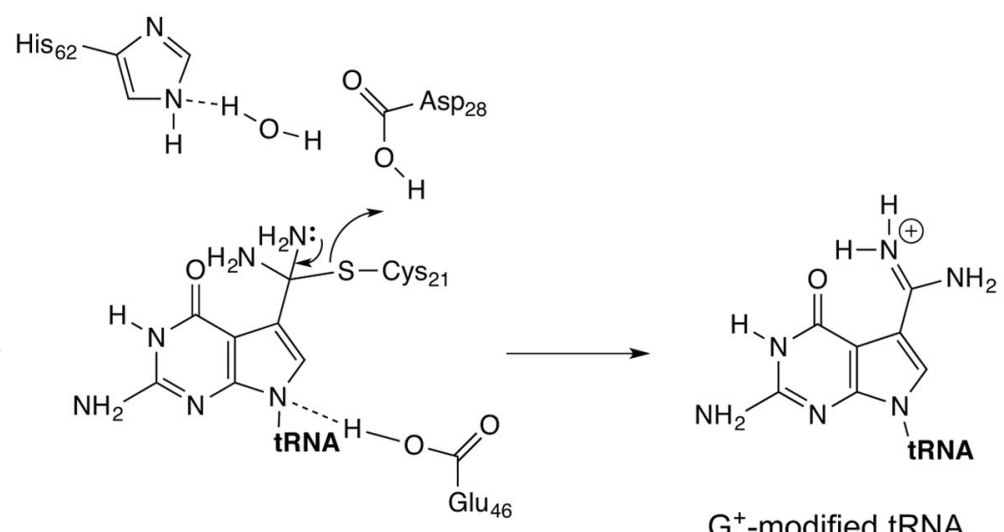

$\mathrm{G}^{+}$-modified tRNA

Figure 8.

Proposed mechanism of QueF-L. 


\section{Table I}

X-ray data collection and structure refinement statistics

\begin{tabular}{|c|c|c|c|c|}
\hline \multicolumn{5}{|l|}{ Data collection } \\
\hline \multicolumn{2}{|l|}{ Space group } & \multicolumn{2}{|l|}{$\mathrm{C} 2$} & $\mathrm{P} 2_{1}$ \\
\hline \multicolumn{2}{|c|}{ Matthew's coefficient $\left(\AA^{3} / \mathrm{Da}\right)$} & \multicolumn{2}{|l|}{2.36} & 2.22 \\
\hline \multicolumn{2}{|l|}{ Solvent content (\%) } & \multicolumn{2}{|l|}{49} & 45 \\
\hline \multicolumn{2}{|l|}{ Monomers/AU } & \multicolumn{2}{|l|}{15} & 10 \\
\hline Dataset & $\mathrm{Se}_{\text {peak }}$ & $\mathrm{Se}_{\text {remote }}$ & $\mathrm{Se}_{\text {inflection }}$ & \\
\hline \multicolumn{5}{|l|}{ Unit cell } \\
\hline a, b, c $(\AA)$ & $215.28,126.73,65.05$ & $215.33,126.78,65.08$ & $215.36,126.81,65.11$ & $62.21,124.99,74.40$ \\
\hline$\beta\left(^{\circ}\right)$ & 102.6 & 102.6 & 102.6 & 112.3 \\
\hline Wavelength $(\AA)$ & 0.97899 & 0.99502 & 0.97953 & 1.12708 \\
\hline Resolution $(\AA)$ & $29-2.74(2.8-2.75)^{1}$ & $29-2.74(2.8-2.75)$ & $29-2.74(2.8-2.75)$ & $69-1.94(1.98-1.94)$ \\
\hline Measured reflections & 334,381 & 333,167 & 328,330 & $4,086,875$ \\
\hline Unique reflections & $43,893(2172)$ & $44,100(2198)$ & $43,527(2160)$ & $77,458(4850)$ \\
\hline Completeness (\%) & $98.7(98.4)$ & $98.7(98.4)$ & $98.7(98.5)$ & $99.8(99.8)$ \\
\hline Multiplicity & $7.6(7.6)$ & $7.6(7.5)$ & $7.5(7.5)$ & $7.4(7.3)$ \\
\hline R-merge $(\%)^{2}$ & $0.060(0.597)$ & $0.052(0.629)$ & $0.060(0.709)$ & $0.049(0.685)$ \\
\hline R-meas ${ }^{3}$ & $0.100(0.753)$ & $0.088(0.780)$ & $0.103(0.903)$ & $0.098(0.66)$ \\
\hline R-pim 4 & $0.036(0.273)$ & $0.032(0.284)$ & $0.037(0.329)$ & $0.052(0.419)$ \\
\hline$\langle\mathrm{I} / \sigma(\mathrm{I})\rangle$ & $25.5(3)$ & $25.4(2.8)$ & $22.9(2.5)$ & $8.1(1.8)$ \\
\hline No. of Bijvoet Pairs & 42,426 & 42,634 & 42,066 & - \\
\hline Anomalous signal & 0.0798 & 0.0687 & 0.0780 & - \\
\hline \multicolumn{4}{|c|}{ FOM after density modification 0.77} & 0.73 \\
\hline \multicolumn{5}{|l|}{ No. of Se sites 17} \\
\hline \multicolumn{5}{|l|}{$\underline{\text { Structure refinement }}$} \\
\hline \multicolumn{2}{|l|}{ Resolution range $(\AA)$} & \multicolumn{2}{|l|}{$29-2.74$} & $69-1.94$ \\
\hline \multicolumn{2}{|c|}{ No. of reflections (working/free) } & \multicolumn{2}{|l|}{$41,291 / 1,975$} & $73,364 / 3,886$ \\
\hline \multicolumn{2}{|c|}{ No. of atoms in the asymmetric unit } & \multicolumn{2}{|l|}{12,487} & 8,338 \\
\hline \multicolumn{2}{|l|}{ Protein } & \multicolumn{2}{|l|}{12,166} & 7,960 \\
\hline \multicolumn{2}{|l|}{ Water } & \multicolumn{2}{|l|}{124} & 379 \\
\hline \multicolumn{2}{|l|}{$\mathrm{PreQ}_{0}$} & \multicolumn{2}{|l|}{195} & - \\
\hline \multicolumn{2}{|l|}{ Sodium ions } & \multicolumn{2}{|l|}{2} & - \\
\hline other & & - & & 3 \\
\hline No. of TLS bodies & & 15 & & 10 \\
\hline $\mathrm{R}_{\text {cryst }} / \mathrm{R}_{\text {free }} 5$ & & $0.20 / 0.25$ & & $0.20 / 0.25$ \\
\hline Deviation from idealit & & & & \\
\hline Bond length $(\AA)$ & & 0.009 & & 0.01 \\
\hline Bond angles $\left({ }^{\circ}\right)$ & & 1.77 & & 1.5 \\
\hline Ramachandran plot- & esidues in: & & & \\
\hline Favored regions $(\%$ & & 96.4 & & 96.7 \\
\hline
\end{tabular}




\begin{tabular}{cll} 
Allowed regions $(\%)$ & 3.0 & 2.1 \\
Outliers $(\%)^{7}$ & 0.6 & 1.2 \\
Wilson B factor $\left(\AA^{2}\right)$ & 49.31 & 34.10 \\
Estimated coordinate error $(\AA)$ & 0.35 & 0.15 \\
Estimated phase error $\left(^{\circ}\right)$ & 28.5 & - \\
\hline
\end{tabular}

${ }^{1}$ Highest-resolution shell information in parentheses.

${ }^{2} \mathrm{R}_{\text {merge }}=\left(\Sigma_{h} \Sigma_{\mathrm{i}}\left|\langle\mathrm{I}(h)\rangle-\mathrm{I}(h)_{\mathrm{i}}\right|\right) / \Sigma_{h} \Sigma_{\mathrm{i}} \mathrm{I}(h) \mathrm{i}$, where $\mathrm{I}(h) \mathrm{i}$ is the $\mathrm{i}^{\text {th }}$ observation of reflection $h$ and $\langle\mathrm{I}(h)\rangle$ is the mean intensity of all observations of reflection $h$.

${ }^{3} \mathrm{R}_{\text {meas }}=\left(\Sigma_{h}\left(\mathrm{n}_{h} /\left(\mathrm{n}_{h}-1\right)\right)^{1 / 2} \Sigma_{\mathrm{i}}|\langle\mathrm{I}(h)\rangle-\mathrm{I}(h) \mathrm{i}|\right) / \Sigma_{h} \Sigma_{\mathrm{i}} \mathrm{I}(h) \mathrm{i}$, where $\mathrm{n}_{h}$ is the number of observations of reflection $h$.

${ }^{4} \mathrm{R}_{\mathrm{pim}}=\left(\Sigma_{h}\left[1 /\left(\mathrm{n}_{h}-1\right)\right]^{1 / 2} \Sigma_{\mathrm{i}}|\langle\mathrm{I}(h)\rangle-\mathrm{I}(h) \mathrm{i}|\right) / \Sigma_{h} \Sigma_{\mathrm{i}} \mathrm{I}(h) \mathrm{i}$.

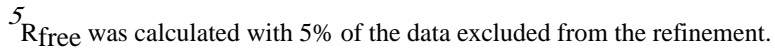

${ }^{6}$ A matrix weighting term of 0.02 was used during refinement in Refmac 5 to tighten the geometry.

7 The residues in disallowed conformations were inspected and are residues followed by a proline rich sequence (e.g., Asn78) or are N-terminal glycine residues. 